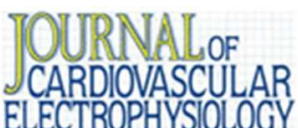

\title{
Mapping and Ablating Ventricular Premature Contractions that Trigger Ventricular Fibrillation: Trigger Elimination and Substrate Modification
}

\begin{tabular}{|r|l|}
\hline Journal: & Journal of Cardiovascular Electrophysiology \\
\hline Manuscript ID: & JCE-140601.R1 \\
\hline Wiley - Manuscript type: & Techniques, Technology, and Innovations \\
\hline Date Submitted by the Author: & n/a \\
\hline Komplete List of Authors: & Nogami, Akihiko; University of Tsukuba, Cardiology \\
\hline & $\begin{array}{l}\text { Clinical: Catheter ablation - ventricular tachycardia, Clinical: Cardiac } \\
\text { mapping - electrogram analysis, Clinical: Electrophysiology - cardiac } \\
\text { arrest/sudden death, Clinical: Non-invasive techniques - Holter/event } \\
\text { recorders, Clinical: Implantable devices - ventricular tachycardia/fibrillation }\end{array}$ \\
\hline &
\end{tabular}


1 "Techniques, Technology, and Innovations in EP" Section in the Journal of the

2 Cardiovascular Electrophysiology

3

$4 \quad$ Mapping and Ablating Ventricular Premature Contractions that Trigger

5 Ventricular Fibrillation: Trigger Elimination and Substrate Modification

6

7 AKIHIKO NOGAMI, M.D., Ph.D.

8 From the Cardiovascular Division, University of Tsukuba, Tsukuba, Ibaraki,

9 Japan

10

11 Brief title: VF Ablation

12

13 Address for correspondence:

14 Akihiko Nogami, M.D., Ph.D., the Cardiovascular Division, University of Tsukuba,

15 1-1-1 Tennodai Tsukuba, Ibaraki, 305-8575, Japan.

16 Tel: $+81-29-853-3142$

17 Fax: $+81-29-853-3143$

18 E-mail: akihiko-ind@umin.ac.jp

20 Abstract: 239 words, Text: 3200 words; 4 figures, 5 references. 
1 Abstract

2 Mapping and Ablating the Trigger of Ventricular Fibrillation.

3 Ventricular fibrillation (VF) is a malignant arrhythmia, usually initiated by a

4 ventricular premature contraction (VPC) during the vulnerable period of

5 cardiac repolarization. Ablation therapy for VF has been described and

6 increasingly reported. Targets for VF triggers are VPC preceded Purkinje

7 potentials or the right ventricular outflow tract (RVOT) in structurally normal

8 hearts, and VPC triggers preceded by Purkinje potentials in ischemic

9 cardiomyopathy. The most important issue before the ablation session is the

10 recording of the 12-lead ECG of the triggering event, which can prove

11 invaluable in regionalizing the origin of the triggering VPC for more detailed

mapping. In cases where the VPC is not spontaneous or inducible, ablation may

be performed by pacemapping. During the session, mapping should be focused

on the earliest activation and determining the earliest potential is the key to a

successful ablation. However, a modification of the Purkinje network might be applied when the earliest site cannot be determined or is located close to the His-bundle. Furthermore, the electrical isolation of the pulmonary artery (PA) can suppress RVOT type polymorphic ventricular tachycardia in some patients with rapid triggers from the PA. Suppression of VF can be achieved by not only the elimination of triggering VPCs, but also by substrate modification of 
1 possible reentry circuits in the Purkinje network, or between the PA and RVOT.

2 Further studies are needed to evaluate the precise mechanisms of this

3 arrhythmia.

4

5 Key words

6 catheter ablation, right ventricular outflow tract, polymorphic ventricular

7 tachycardia, Purkinje network, trigger beat, ventricular fibrillation

8

9 


\section{Introduction}

2

3

4

5 idiopathic VF could be suppressed by catheter ablation of those triggers

6 originating from the Purkinje system or right ventricular outflow tract (RVOT)

7 and the ablation therapy for VF has been increasingly reported during the last

8 decade. In general, this ablation appears to have a high success rate and is

9 relatively easy to perform, although precise mapping is required. However,

little is known about the initiating mechanism of VF. Further, whether the mechanism of the ablation effect is due to the suppression of the trigger or substrate modification is also unclear. The objective of this review was to summarize the strategies we have incorporated into our catheter ablation procedures for VF, especially in difficult and complicated cases.

\section{Twelve-lead Recording of Triggering VPCs}

Most cases of VF appear to originate from the Purkinje system, and some cases report initiating events that are distinct from the cardiac conduction system such as the RVOT [1]. Recording of the 12-lead ECG of the triggering event can prove invaluable in regionalizing the origin of the triggering VPC for 
1 more detailed mapping, and an effort to record such a trigger should be

2 routine. The target site can be speculated with the 12-lead ECG

3 documentation: RVOT, right distal Purkinje, left posterior Purkinje, or left

4 anterior Purkinje system. In the patients without ectopy, the putative source of

5 the VPC can be ablated in sinus rhythm based on pace mapping followed by

6 radiofrequency (RF) energy delivery. In the patients with multifocal VPCs, the

7 true triggering VPC that initiates VF or nonsustained polymorphic ventricular

8 tachycardia (VT) has to be confirmed. It is essential that there is accurate

9 documentation of the triggering VPC, with a 12-lead ECG.

Figure 1 shows ECGs from a 59-year-old female patient with early

11 repolarization associated with VF [2]. Each panel shows the QRS complexes

12 during sinus rhythm and the VPC. In the emergency room, significant J-ST elevation in the infero-lateral leads and VPC bigeminy with a right bundle branch block (RBBB) configuration and superior axis were observed after the spontaneous termination of polymorphic ventricular tachycardia (VT) (Fig. 1A).

One month after the implantation of a defibrillator (ICD), a triggering VPC ablation was performed due to frequent ICD shocks. During the ablation session, frequent monofocal VPCs were observed (Fig. 1B), and Purkinje potentials on the posterior left ventricular septum preceded the onset of the VPC by $65 \mathrm{~ms}$. An RF energy application at that site immediately eliminated the 
1 VPC. However, a few days after the session, VF recurred. A 12-lead Holter

2 recording could record the initiation of the VF (Fig. 1C). The "true" triggering

3 VPC was similar to the ablated VPC, but different (especially lead aVR).

4 Interestingly, while J-ST elevation was recorded in the emergency room and

5 during the VF recurrence, it was not observed during the ablation session.

6 There was a possibility that the true triggering VPC appeared only during the J-

7 ST elevation. The patient did not prefer to undergo a re-ablation session and

8 the oral administration of disopyramide successfully suppressed the VF

9 recurrence.

10 In the intensive care unit, a synthesized 12-lead ECG from the signals

11 recorded using three to five electrodes is sometimes used. In our experience,

12 the limb leads in the synthesized 12-lead ECG are similar to the Mason-Likar

13 lead configuration, in which the limb lead electrodes are placed on the torso

14 rather than the distal extremities, and can be used for the morphology analysis

15 of VPCs. However, the chest lead information in the synthesized ECG is less

16 useful because of its inaccuracy. Twelve-lead Holter monitoring also uses a

17 Mason-Likar lead configuration similar to the limb leads and the real six chest

18 electrodes for the chest leads, and appears to be highly reliable and useful for

19 the diagnosis of "true" triggering VPCs. 


\section{Substrate Modification of the Purkinje Network}

2

3

4

5 targeting the Purkinje potentials responsible for triggering VF has been shown

6 to be possible and efficacious in a number of conditions such as idiopathic VF

7 (short-coupled variant of torsade de pointes), ischemic VF, and chronic

8 myocarditis. What is still undetermined is whether the mechanism of the

9 ablation effect is due to the suppression of the trigger or substrate modification.

During activation mapping of the triggering VPC, attention should be paid to the preceding sharp Purkinje-like signals. Mapping should be focused on the earliest activation of this potential, and determining the earliest potential is the key to a successful ablation. However, the potential may sometimes be seen to occur with intra-Purkinje block to the myocardium, and not produce a VPC.

This means that there is the possibility that not only the elimination of the triggering VPC, but also conduction block in the Purkinje network can suppress the triggering VPC and VF. In fact, dissociated firing from the Purkinje network is sometimes seen after a successful ablation. The following case is an example of the successful suppression of VF by the modification of the Purkinje network 
$1 \quad[3]$.

2

3

4

5 polymorphic VT was repeatedly inducible by atrial pacing after an intravenous

6 administration of cibenzoline (Fig. 2A). There was no change in the QRST

7 complexes in any of the electrograms after the intravenous administration of

8

9 cibenzoline. The first VPC (VPC1) had an RBBB configuration with right-axis deviation and the second one (VPC2) had an RBBB pattern with a northwest axis. The coupling interval of VPC1 to the preceding normally conducted QRS complex was 250 ms. During the polymorphic VT, diastolic and presystolic Purkinje potentials were recorded from an octapolar electrode catheter with 1.25-mm electrode widths and 2-mm inter-electrode spacings placed on the left ventricular septum (Fig. 2A and Fig. 2B). Diastolic Purkinje potentials were recorded earlier from the proximal than distal electrodes, and fused presystolic Purkinje potentials were recorded earlier from the distal than proximal electrodes. During sinus rhythm, recording at the same site demonstrated fused Purkinje potentials before the onset of the QRS. Because the earliest Purkinje activation site before VPC1 could not be determined and seemed to be a more proximal site than the site of electrodes 7-8, RF energy was 
1 delivered to the site of electrodes 3-4. A Purkinje potential from this site

2 preceded the onset of VPC1 by $15 \mathrm{~ms}$ and VPC2 by $60 \mathrm{~ms}$. The intracardiac

3 electrograms recorded after the ablation showed the abolition of the local

4 Purkinje potentials at the middle portion and a slight delay in the occurrence of

5 the local ventricular electrogram during sinus rhythm (Fig. 2C). The

6 polymorphic VT became noninducible and only an isolated VPC was inducible.

7 The morphology of this isolated VPC differed from the previous triggering VPCS

8 (VPC1 or VPC2). Further, Purkinje firing was observed before this VPC and

9 intra-Purkinje block occurred. Holter monitoring after the ablation revealed no

VPCs. He was followed up without any drugs or episodes of syncope or VF

11 recurrences during a follow-up period of 14 years. These observations suggest

12 that the VF initiation was caused by activity from the Purkinje tissue. However,

13 the suppression of the VF was achieved with catheter ablation of the Purkinje

network, not of the earliest Purkinje activation of the initial triggering beat in

this patient. If the early phase of VF is perpetuated by variable reentrant loops

within the Purkinje network, the mechanism of VF suppression in this patient

can be explained by intra-Purkinje block. 
1 determine how much of the complex Purkinje network was involved in each

2 patient and the issue of multiple foci versus differing activation routes from

3 limited foci remains unsolved. In our case, catheter mapping revealed that the

4 constantly changing polymorphic QRS morphology resulted from the changing

5 propagation in the Purkinje arborization and the polymorphic VT became

6 noninducible after the catheter ablation of the Purkinje network. We did not

7 ablate the earliest site of the Purkinje activation, and the isolated VPC with

8 diastolic Purkinje activation was still inducible after the catheter ablation.

9 Of course, the earliest activation site of the Purkinje activation during the

10 triggering VPC should be searched and ablated; however, a modification of the

11 Purkinje network might be applied when the earliest site cannot be

12 determined or is located close to the His-bundle. In my experience, the right-

13 sided triggers usually arise from the distal right bundle branch and the most

14 proximal site of the origins on the left side was the bifurcation of the left

15 anterior and posterior fascicles. If the earliest site is located proximal to the

16 bifurcation, ablation of just the distal site is recommended for the initial

17 application. It is possible to create substrate modification and eliminate the

18 origin nearby because the Purkinje network can be easily ablated.

Because the Purkinje network in humans is mostly localized to the

subendocardium, a transmural lesion creation is not needed. Further, the 
1 ventricular myocardium of the culprit Purkinje network in idiopathic VF is

2 usually healthy. This differs from ischemic VF, in which the ventricular

3 myocardium at the culprit Purkinje network usually has a low-voltage and is

4 located near a scar-border. During the Purkinje network modification, the

5 creation of bundle-branch block or hemi block is not required. While some

6 change in the frontal axis has been observed in some patients after the

7 ablation during a left septal Purkinje ablation, the QRS width remains almost

8 the same. Catheter manipulation sometimes produces transient bundle-branch

9 block. As a result, peripheral Purkinje potentials no longer precede the local

10 ventricular activation in sinus rhythm, and it make mapping of the Purkinje

11 network difficult. For this reason the creation of bundle branch block should be

12 avoided.

\section{Substrate Modification for RVOT Type Polymorphic VT}

The RVOT is the most common origin of monomorphic VT in structurally normal hearts and it is also the origin for triggers of polymorphic VT, which

17 rapidly degenerates into VF. This type of ablation is essentially no different

18 than the ablation of idiopathic RVOT-VPCs or VT. The ablation targets the site

19 of earliest activation and pacemapping in the RVOT [4]. The following case 
1 demonstrates an unusual patient with the suppression of polymorphic VT by

2 conduction block between the pulmonary artery (PA) and the RV.

3

4

5 VT (Fig. 3A). The mean cycle length of the VT was $220 \mathrm{~ms}$ and the morphologies

6 of the first three QRS complexes of the polymorphic VT were always the same.

7 Electroanatomical mapping was performed and the propagation map of the

8 first VPC had a centrifugal pattern from the posterior attachment of the RVOT.

9 From that site, pace mapping was performed. Interestingly, pacing at a cycle length of 300 ms created the exact same polymorphic QRS configurations as those during the clinical polymorphic VT (Fig. 3B and Fig. 3C). After several RF energy applications to the posterior RVOT, the repetitive VPCs disappeared. However, isolated VPCs with a slightly different QRS morphology and longer coupling interval remained. Therefore, mapping in the PA was performed. From the PA, a delayed PA potential was recorded during sinus rhythm and that potential preceded the onset of the QRS during the VPC that remained (Fig. 4A). Between the PA and RVOT potentials, a tiny bridging potential was recoded. RF energy was delivered at this site in the PA. Just after the RF energy application, the PA potential disappeared (Fig. 4B). Repetitive firing from the PA was observed; however, there were no VPCs. These findings indicate there 
1 was bidirectional conduction block between the PA and RVOT. In this case, the

2 site-of-origin of the triggering beat was in the PA, and the multiple exits or non-

3 uniform conduction to the RVOT might create the polymorphism of the VT. In

4 fact, the change in the QRS configuration was reproduced by pacing at a

5 relatively long cycle length. In this case, the electrical isolation of the

6 extracardiac vessel, i.e. PA, suppressed the fibrillatory arrhythmia in the

7 connecting heart chamber, i.e. RV. Interestingly, it is quite similar to the

8 relationship between the pulmonary vein and left atrium in the mechanism of

9 paroxysmal atrial fibrillation.

It has now been clearly established that myocardial sleeves extend into the great arteries for variable distances. These myocardial sleeves commonly extend fairly symmetrically crossing each of the 3 pulmonary valve cusps. The extensions can vary from a few $\mathrm{mm}$ up to more than $2 \mathrm{~cm}$ into the pulmonary artery [5]. The outflow tract artery junction is complex both in terms of its development and histologically with multiple tissue types interfacing in this region. The precise mechanism of the polymorphic changes in the QRS complex cannot be clarified from our results. However, based on our results [4] and previously reported data [5], the functional block or delayed conduction by rapid firing due to triggered activity or micro-reentry arising from a single focus led to chaotic conduction, causing polymorphic VT/VF without an organic 
1 delayed conduction zone. In the presented case, burst pacing from the earliest

2 activation site could reproduce several initial QRS complexes identical to the

3 documented polymorphic VT. This reproducibility suggested that the

4 polymorphic VT from the RVOT occurred from a single focus by triggered

5 activity or micro-reentry with multiple myocardial exits to the RV and the

6 development of polymorphic QRS waves. In addition, we recently

7 demonstrated the shorter coupling interval $(\mathrm{Cl})$ index $(\mathrm{Cl} /$ preceding $\mathrm{R}-\mathrm{R})$ in

8 the patients with polymorphic VT than in those with monomorphic VT and the

9 shorter $\mathrm{Cl}$ index during VT than isolated VPCs in the same patients with

10 polymorphic VT [4]. These might result from complexes that are impinging on

11 the ventricular refractoriness (producing dispersion of refractoriness) or may

12 be related to a triggered mechanism of initiation. In the presented case, the

13 repetitive VPCs disappeared after several RF applications to the posterior RVOT.

14 A residual isolated VPC had a slightly different QRS morphology and longer $\mathrm{Cl}$

15 and originated from the PA.

17 Conclusion

VF is a lethal arrhythmia that may be present in patients with or without

19 structural heart disease. RF catheter ablation of VF is feasible and can be used 
1 be achieved by not only the elimination of triggering VPCs, but also the

2 creation of conduction block between the PA and RVOT, or of the Purkinje

3 network. Further studies are needed to evaluate the precise mechanisms of

4 this arrhythmia.

5 


\section{1 \\ References}

2

3

4

5

1. Haïssaguerre M, Shoda M, Jais P, Nogami A, Shah DC, Kautzner J, Arentz T, Kalushe D, Lamaison D, Griffith M, Cruz F, Paola A, Gaïta F, Hocini M, Garrigue S, Macle L, Weerasooriya R, Clementy J: Mapping and ablation of idiopathic ventricular fibrillation. Circulation 2002; 106; 962-967.

2. Haïssaguerre M, Derval N, Sacher F, Jesel L, Deisenhofer I, de Roy L, Pasquié J-L, Nogami A, Babuty D, Yli-Mayry S, De Chillou C, Scanu P, Mabo P, Matsuo S, Probst V, Le Scouarnec S, Defaye P, Schlaepfer J, Rostock T, Lacroix D, Lamaison D, Lavergne T, Aizawa Y, Englund A, Anselme F, O'Neill M, Hocini M, Lim KT, Knecht S, Veenhuyzen GD, Bordachar P, Chauvin M, Jais P, Coureau G, Chene G, Klein GL, Clémenty J: Sudden cardiac arrest associated with early repolarization. N Eng J Med 2008, 358: 2016-2023.

3. Nogami A, Sugiyasu A, Kubota S, Kato K: Mapping and ablation of idiopathic ventricular fibrillation from Purkinje system. Heart Rhythm 2005; 2: 646649.

4. Igarashi M, Tada H, Kurosaki K, Yamasaki H, Akiyama D, Sekiguchi Y, Kuroki K, Machino T, Murakoshi N, Nakata Y, Kuga K, Nogami A, Aonuma K:

Electrocardiographic Determinants of the Polymorphic QRS Morphology in Idiopathic Right Ventricular Outflow Tract Tachycardia. J Cardiovasc Electrophysiol 2012; 23: 521-526. 
1 5. Asirvatham SJ: Correlative anatomy for the invasive electrophysiologist:

2 outflow tract and supravalvar arrhythmia. J Cardiovasc Electrophysiol 2009;

$3 \quad \underline{20: 955-968}$

4 


\section{$1 \quad$ Figure Legends}

2 Figure 1. Surface 12-lead ECGs from a female patient with early repolarization

3

4

5 lateral leads; and VPC bigeminy with an RBBB configuration and superior axis

$6 \quad$ were observed after the spontaneous termination of polymorphic ventricular

7 tachycardia (VT). (B) During the ablation session, frequent monofocal VPCs

8 with an RBBB configuration and superior axis were observed. (C) A 12-lead

9 Holter recording could record the VF recurrence. The "true" triggering VPC is

10 similar to the ablated VPC, but is different (especially lead aVR) (arrowhead).

11 Interestingly, while J-ST elevation was recorded in the emergency room and

12 during the VF recurrence (arrows), it was not observed during the ablation

13 session.

Figure 2. Catheter mapping during polymorphic VT in a male patient with a short-coupled variant of torsade de pointes [3].

(A) During the polymorphic VT which was induced by rapid atrial pacing after the administration of intravenous cibenzoline, diastolic Purkinje potentials and presystolic Purkinje potentials were recorded from the left 
1 ventricular septum. During sinus rhythm, fused Purkinje potentials were

2 recorded before the onset of the QRS. (B) Representation of an octapolar

3 electrode catheter placed on the left ventricular septum. (C) Intracardiac

4 electrograms recorded after ablation showing the abolition of the local

5 Purkinje potential (P) at the middle portion and a slight delay in the occurrence

6 of the local ventricular electrogram during sinus rhythm (arrow). The

7 polymorphic VT became noninducible and only an isolated VPC was inducible.

8 The morphology of this VPC differed from the previous triggering VPC and

9 intra-Purkinje block was also observed before this VPC (arrowhead).

$\mathrm{HBE}=$ His-bundle electrogram; HRA = high right atrium; $\mathrm{LAO}=$ left

anterior oblique view; $\mathrm{LV}=$ left ventricle; $\mathrm{P}=$ Purkinje potential; $\mathrm{RAO}=$ right

anterior oblique view; $S_{A}=$ atrial pacing stimulus. (From Nogami $A$, Sugiyasu $A$,

Kubota S, et al. Mapping and ablation of idiopathic ventricular fibrillation from

Purkinje system. Heart Rhythm 2005, 2: 646-649. With permission.)

Figure 3. Surface 12-lead ECGs in a female patient with an RVOT type polymorphic VT.

(A) Holter monitoring revealed frequent episodes of polymorphic VT. The mean cycle length of the VT was $220 \mathrm{~ms}$ and the morphologies of the first 
1 three QRS complexes of the polymorphic VT were always the same. (B)

2 Polymorphic VPC couplets were recorded during the ablation session. (C)

3 Pacemapping at the earliest activation site in the RVOT reproduced the exact

4 same polymorphic QRS configurations as those during the clinical polymorphic

5 VT.

6

7

8 Figure 4. Successful ablation in the pulmonary artery.

9 (A) From the pulmonary artery (PA), a delayed PA potential was recorded during sinus rhythm (arrow head) and this potential preceded the onset of the QRS during the remaining VPC (arrow). Between the PA and RVOT potentials, a tiny bridging potential was recorded. (B) Just after the RF energy application in the PA, the PA potential (arrow heads) disappeared. Repetitive artery potential; RF = radiofrequency energy. 
Figure 1. Surface 12-lead ECGs from a female patient with early repolarization associated with VF. (A) In the emergency room, significant J-ST elevation in the infero-lateral leads and VPC bigeminy with an RBBB configuration and superior axis were observed after the spontaneous termination of polymorphic ventricular tachycardia (VT). (B) During the ablation session, frequent monofocal VPCs with an RBBB configuration and superior axis were observed. (C) A 12-lead Holter recording could record the VF recurrence. The "true" triggering VPC is similar to the ablated VPC, but is different (especially lead aVR) (arrowhead). Interestingly, while J-ST elevation was recorded in the emergency room and during the VF recurrence (arrows), it was not observed during the ablation session. 
A

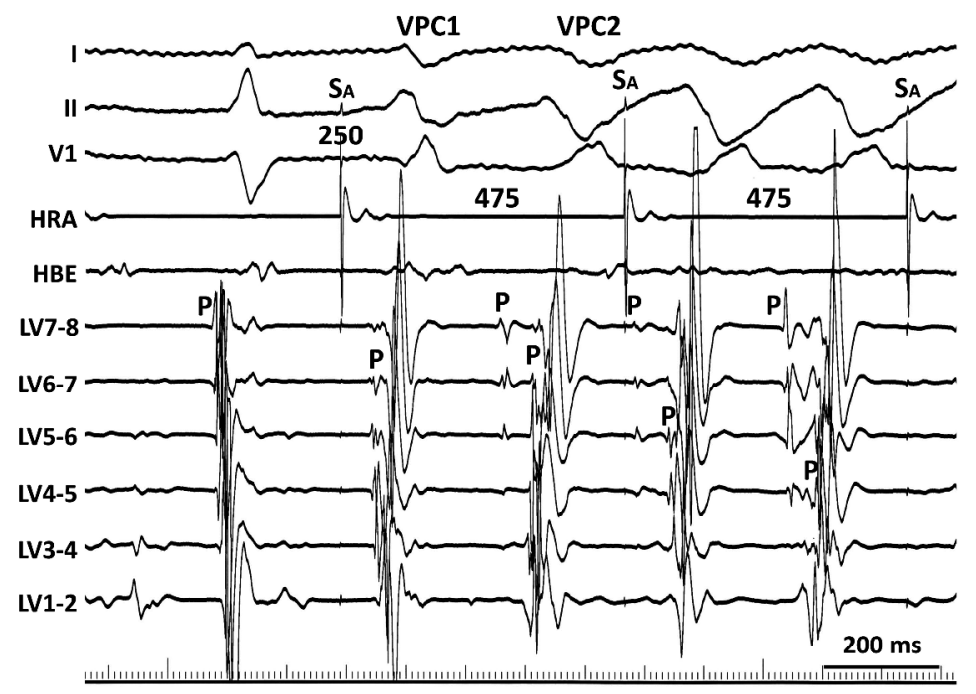

B
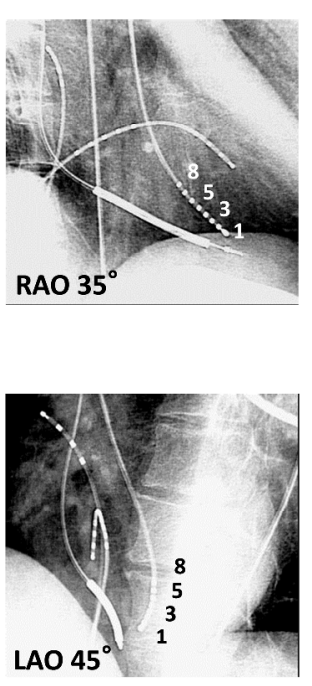

Figure 2AB. Catheter mapping during polymorphic VT in a male patient with a short-coupled variant of torsade de pointes [3].

(A) During the polymorphic VT which was induced by rapid atrial pacing after the administration of intravenous cibenzoline, diastolic Purkinje potentials and presystolic Purkinje potentials were recorded from the left ventricular septum. During sinus rhythm, fused Purkinje potentials were recorded before the onset of the QRS. (B) Representation of an octapolar electrode catheter placed on the left ventricular septum. $1719 \times 1190 \mathrm{~mm}$ (96 × 96 DPI) 

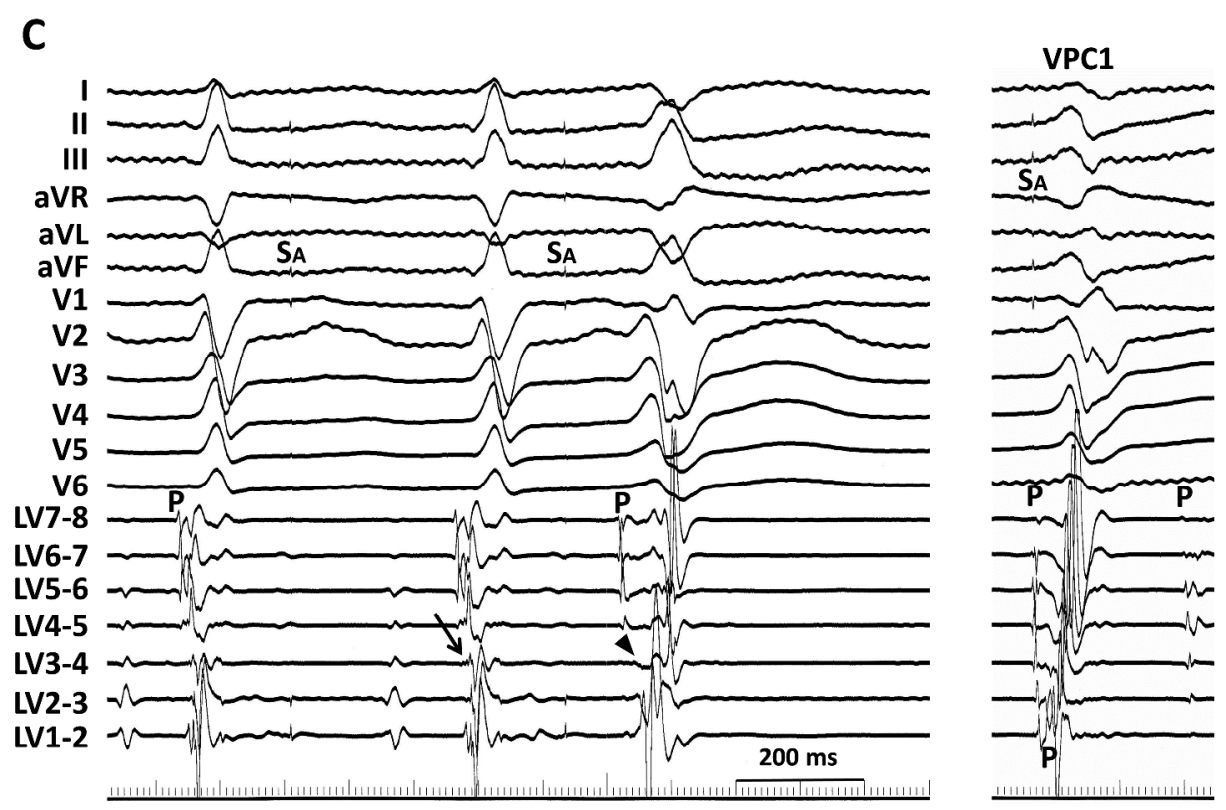

Figure 2C. Catheter mapping during polymorphic VT in a male patient with a short-coupled variant of torsade de pointes [3].

(C) Intracardiac electrograms recorded after ablation showing the abolition of the local Purkinje potential (P) at the middle portion and a slight delay in the occurrence of the local ventricular electrogram during sinus rhythm (arrow). The polymorphic VT became noninducible and only an isolated VPC was inducible. The morphology of this VPC differed from the previous triggering VPC1 and intra-Purkinje block was also observed before this VPC (arrowhead).

$\mathrm{HBE}=$ His-bundle electrogram; HRA = high right atrium; $\mathrm{LAO}=$ left anterior oblique view; $\mathrm{LV}=$ left ventricle; $\mathrm{P}=$ Purkinje potential; $\mathrm{RAO}=$ right anterior oblique view; $\mathrm{SA}=$ atrial pacing stimulus. (From Nogami A, Sugiyasu A, Kubota S, et al. Mapping and ablation of idiopathic ventricular fibrillation from Purkinje system. Heart Rhythm 2005, 2: 646-649. With permission.) 


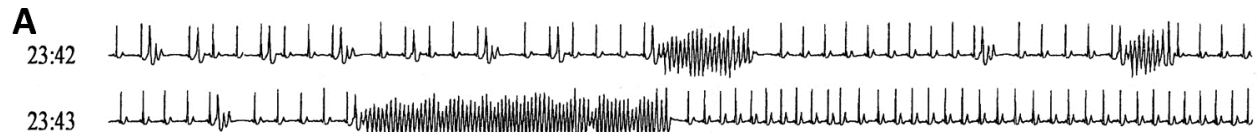

B

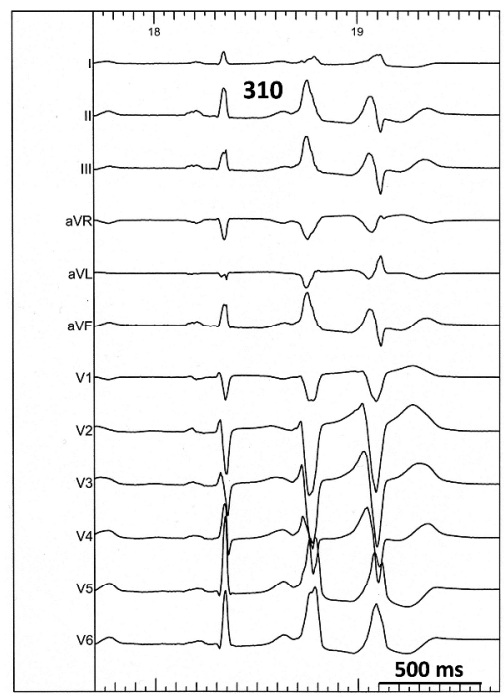

$10 \mathrm{~s}\lfloor\perp \perp \perp \perp \perp \mid \perp \perp\rfloor$

c

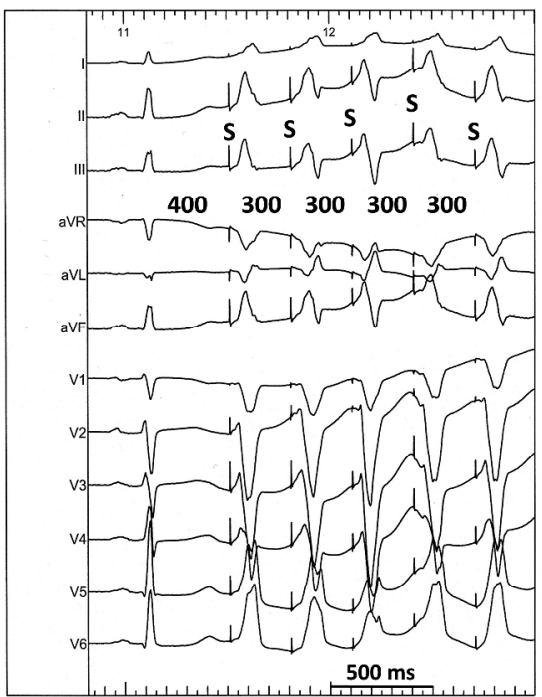

Figure 3. Surface 12-lead ECGs in a female patient with an RVOT type polymorphic VT.

(A) Holter monitoring revealed frequent episodes of polymorphic VT. The mean cycle length of the VT was $220 \mathrm{~ms}$ and the morphologies of the first three QRS complexes of the polymorphic VT were always the same. (B) Polymorphic VPC couplets were recorded during the ablation session. (C) Pacemapping at the earliest activation site in the RVOT reproduced the exact the same polymorphic QRS configurations as those during the clinical polymorphic VT. 
A

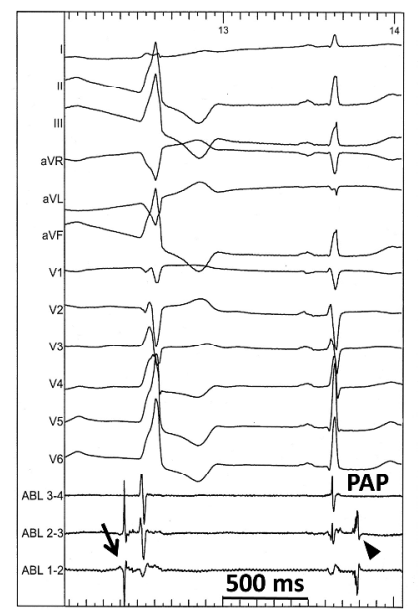

B

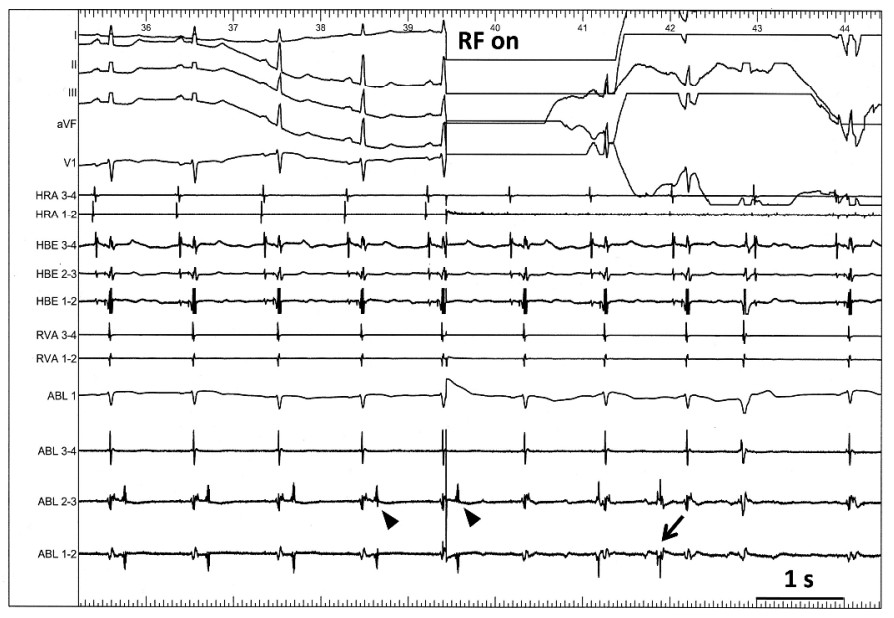

Figure 4. Successful ablation in the pulmonary artery.

(A) From the PA, a delayed PA potential was recorded during sinus rhythm (arrow head) and this potential preceded the onset of the QRS during the remaining VPC (arrow). Between the PA and RVOT potentials, a tiny bridging potential was recorded. (B) Just after the RF energy application in the PA, the PA potential (arrow heads) disappeared. Repetitive firing from the PA was observed (arrow); however, there were no VPCs. These findings indicate that bidirectional conduction block occurred between the PA and RVOT. $\mathrm{HBE}=$ His-bundle electrogram; HRA = high right atrium; PAP = pulmonary artery potential; RF = radiofrequency energy. $1719 \times 1190 \mathrm{~mm}(96 \times 96 \mathrm{DPI})$ 